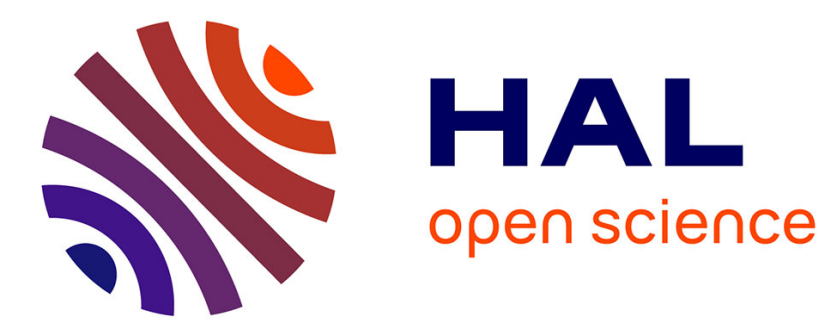

\title{
A one-parameter deformation of the noncommutative Lagrange inversion formula
}

\author{
Jean-Paul Bultel
}

\section{To cite this version:}

Jean-Paul Bultel. A one-parameter deformation of the noncommutative Lagrange inversion formula. International Journal of Algebra and Computation, 2011, pp.1395-1414. hal-00825466

\section{HAL Id: hal-00825466 https://hal.science/hal-00825466}

Submitted on 23 May 2013

HAL is a multi-disciplinary open access archive for the deposit and dissemination of scientific research documents, whether they are published or not. The documents may come from teaching and research institutions in France or abroad, or from public or private research centers.
L'archive ouverte pluridisciplinaire HAL, est destinée au dépôt et à la diffusion de documents scientifiques de niveau recherche, publiés ou non, émanant des établissements d'enseignement et de recherche français ou étrangers, des laboratoires publics ou privés. 


\title{
A ONE-PARAMETER DEFORMATION OF THE NONCOMMUTATIVE LAGRANGE INVERSION FORMULA
}

\author{
JEAN-PAUL BULTEL
}

\begin{abstract}
We give a one-parameter deformation of the noncommutative Lagrange inversion formula, more precisely, of the formula of Brouder-Frabetti-Krattenthaler for the antipode of the noncommutative Faá di Bruno algebra. Namely, we obtain a closed formula for the antipode of the one-parameter deformation of this Hopf algebra discovered by Foissy.
\end{abstract}

\section{INTRODUCTION}

The existence of combinatorial interpretations of the Lagrange inversion formula [12] can be traced back to the existence of noncommutative generalizations $[4,11]$. In its simplest form, the classical version gives the compositional inverse of an invertible formal power series. In other words, it expresses the antipode of the Hopf algebra of polynomial functions on the group of formal diffeomorphisms of the real line, also known as the Faá di Bruno algebra [5].

Formal power series in one variable with coefficients in a noncommutative algebra can be composed (by substitution of the variable). This operation is not associative, so that they do not form a group. However, the analogue of the Faá di Bruno algebra still exists in this context. It is investigated in [1] in view of applications in quantum field theory. In [1], one finds in particular a combinatorial formula for its antipode. This formula is rederived by Novelli and Thibon [10], who also show that it is equivalent to the noncommutative Lagrange formula of Gessel and Pak-Postnikov-Retakh. They obtain it from the Brouder-Frabetti-Krattenthaler formula by a simple application of the antipode of the Hopf algebra of noncommutative symmetric functions.

In [2], Foissy obtains, as a byproduct of his investigation of combinatorial SchwingerDyson equations, one-parameter families of Hopf algebras. They interpolate respectively between symmetric functions and Faá di Bruno, and between noncommutative symmetric functions and the noncommutative Faá di Bruno algebra.

The main result of this paper is a closed formula for the antipode of the noncommutative family. As we shall see, this is a natural deformation of the Brouder-FrabettiKrattenthaler formula. As in the original version, we obtain a closed formula for the antipode of the simple complete noncommutative symmetric functions $S_{n}$. Namely, we compute explicitely the coefficients $\alpha_{I}(I \vDash n)$ in their expansion in the basis of complete noncommutative symmetric functions $S^{I}$. As in the original version, we

Date: May 17, 2011. 
end up with a sum of products of binomial coefficients over a Catalan set, that is

$$
\alpha_{I}=(-1)^{l(I)} \sum_{\rho \in \mathcal{A}_{l(I)}} \prod_{k=1}^{l(I)}\left(\begin{array}{c}
i_{k} \gamma+1 \\
\rho_{k}
\end{array}\right),
$$

where $\mathcal{A}_{n}$ is the set of sequences $\rho=\left(\rho_{1}, \rho_{2}, \ldots, \rho_{n}\right)$ of length $n$, where the $\rho_{i}$ are nonnegative integers such that

$$
\left\{\begin{array}{cccccc}
\rho_{1} & +\ldots & + & \ldots & + & \rho_{n}=n-1 \\
& \rho_{2} & + & \ldots & + & \rho_{n} \leq n-2 \\
& & \ddots & & & \vdots \\
& & & & & \rho_{n} \leq 0
\end{array}\right.
$$

We also give a recurrence formula for the antipode of the simple complete noncommutative symmetric functions. Namely, we obtain a formula for their expansion in the ribbon basis, and some other properties of the corresponding coefficients.

\section{NotATIONS AND BACKGROUND}

2.1. A one-parameter family of Hopf algebras. We denote by Sym the Hopf algebra of noncommutative symmetric functions. Our notations for noncommutative symmetric functions will be as in $[3,6]$.

In this text, we will be interested in a deformation $\mathcal{H}_{\gamma}$ of the algebra $\mathcal{H}$ of noncommutative formal diffeomorphisms, where $\gamma$ is a real parameter. As an associative algebra, $\mathcal{H}_{\gamma}$ coincides with the algebra of noncommutative symmetric functions.

Its coproduct $\Delta_{\gamma}$ is given on complete symmetric functions by the formula

$$
\Delta_{\gamma} S_{n}(A)=\sum_{k=0}^{n} S_{k}(A) \otimes S_{n-k}((k \gamma+1) A)
$$

where for a scalar $\alpha, S_{n}(\alpha A)$ is defined as the coefficient of $t^{n}$ in

$$
\sigma_{t}(\alpha A)=\sigma_{t}(A)^{\alpha}=\left(\sum_{n \geq 0} t^{n} S_{n}(A)\right)^{\alpha}
$$

(see [6]). This deformation of the noncommutative Faà di Bruno Hopf algebra of [1] has been recently discovered by Foissy ([2]) in his investigation of combinatorial Dyson-Schwinger equations in the Connes-Kreimer algebra. As a Hopf algebra, $\mathcal{H}_{0}$ is the algebra of noncommutative symmetric functions, and the noncommutative Faà di Bruno Hopf algebra is the case $\gamma=1$. It can be shown that for $\gamma \neq 0, \mathcal{H}_{\gamma}$ is isomorphic to $\mathcal{H}_{1}[2]$.

2.2. Conventions. We denote by $A$ the underlying alphabet of the standard realization of noncommutative symmetric functions. We identify any $F \in \mathbf{S y m}$ with its realization $F(A)$ when convenient.

We denote by $F \mapsto F^{\star}$ the antipode of $\mathcal{H}_{\gamma}$ and we set $X=\sigma_{1}(A)^{\star}$. We denote by $X_{k}=S_{k}^{\star}$ the $k$ th homogeneous component of $X$, and for any composition

$$
I=\left(i_{1}, i_{2}, \ldots, i_{r}\right)
$$


we set $X^{I}=X_{i_{1}} X_{i_{2}} \cdots X_{i_{r}}$. For

$$
F=\sum_{I} a_{I} S^{I}
$$

we set $F_{\mid I}=a_{I} S^{I}$.

For $J=\left(j_{1}, j_{2}, \ldots\right) \vDash n$ and $I=\left(i_{1}, i_{2}, \ldots\right) \vDash l(J)$, we set

$$
C_{I}(J)=\left(j_{1}+\ldots+j_{i_{1}}, j_{1+i_{1}}+\ldots+j_{i_{1}+i_{2}}, j_{i_{1}+i_{2}+1}+\ldots+j_{i_{1}+i_{2}+i_{3}}, \ldots\right)
$$

One has $C_{I}(J) \vDash n$ and $l\left(C_{I}(J)\right)=l(I)$. Set also

$$
\mathfrak{C}(J)=\left\{C_{I}(J) \mid I \models l(J)\right\},
$$

and denote by $\mathcal{A}_{n}$ the set of sequences $\rho=\left(\rho_{1}, \rho_{2}, \ldots, \rho_{n}\right)$ of length $n$, where the $\rho_{i}$ are nonnegative integers such that

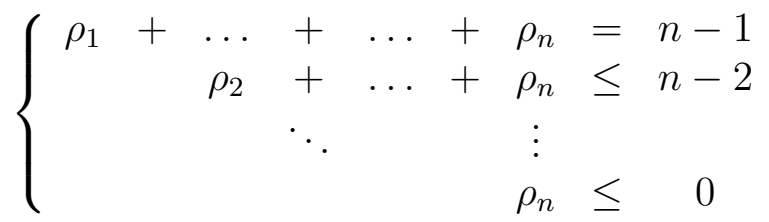

We reserve the letters $I, J$ and $K$ to denote compositions of integers. As we shall never mention integer partitions in this document, we use the letters $\lambda, \mu, \nu$ and $\rho$ to denote elements of the sets $\mathcal{A}_{n}$. For any finite sequence $\rho$ of integers, we denote by $|\rho|$ the sum of its elements and by $l(\rho)$ its length.

\section{A Closed formula for the antipode of the Hopf algebra $\mathcal{H}$ of FORMAL DIFFEOMORPHISMS}

The classical Lagrange inversion formula for the reversion of formal power series can be interpreted in terms of classical symmetric functions (see [9], Ex. 24 p. 35, Ex. 25 p. 132, [7] Section 2.4 and [8]). Similarly, for various noncommutative analogues of the Lagrange inversion formula (see [4], [11] and [1]), Novelli and Thibon give in [10] interpretations in terms of noncommutative symmetric functions.

Brouder, Frabetti and Krattenthaler obtain in [1] a form of the noncommutative Lagrange inversion formula. Namely, they give an explicit formula for the antipode of the Hopf algebra $\mathcal{H}$ of noncommutative formal diffeomorphisms. The elements of $\mathcal{H}$ can be identified with noncommutative symmetric functions by means of the correspondence $a_{n}$ (of [1]) $=S_{n}$ (of Sym), which identifies $\mathcal{H}$ with Sym as an associative algebra. Under this correspondence, the coproduct $\Delta$ on $\mathcal{H}$ takes the form

$$
\Delta S_{n}(A)=\sum_{k=0}^{n} S_{k}(A) \otimes S_{n-k}((k+1) A)
$$

We denote again by $\star$ the corresponding antipode. Novelli and Thibon give in [10] a combinatorial interpretation for the coefficient $\lambda_{I}$ defined by

$$
S_{n}^{\star}=\sum_{I \models n}(-1)^{l(I)} \lambda_{I} S^{I},
$$


whose value is given in [1] by

$$
\lambda_{I}=\sum_{\left(a_{1}, \ldots, a_{p-1}\right)} \prod_{k=1}^{p-1}\left(\begin{array}{c}
i_{k}+1 \\
a_{k}
\end{array}\right)
$$

In formula (12), the sum is taken over the set of all the sequences $\left(a_{1}, \ldots, a_{p-1}\right)$ where the $a_{i}$ are nonnegative integers such that

$$
a_{1}+\ldots+a_{p-1}=p-1
$$

and for all $1 \leq j<p-1$,

$$
a_{1}+\ldots+a_{j} \leq j
$$

Now, let us explain this combinatorial interpretation. We have

$\Delta\left(\sigma_{1}(A)\right)=\sum_{n \geq 0} \sum_{k=0}^{n} S_{k}(A) \otimes S_{n-k}((k+1) A)=\sum_{k \geq 0} S_{k}(A) \otimes \sigma_{1}((k+1) A)=\sum_{k \geq 0} S_{k} \otimes \sigma_{1}(A)^{k+1}$,

so that

$$
1=\sum_{k \geq 0} S_{k}\left(S_{0}^{\star}+S_{1}^{\star}+S_{2}^{\star}+\ldots\right)^{k+1}
$$

Denote by $h$ the sum of all $h_{k}=S_{k}^{\star}$ with $k \geq 0$. This formula can be rewritten as

$$
h^{-1}=S_{0}+S_{1} h+S_{2} h^{2}+\ldots
$$

Setting $c=S_{0}^{-1}$ and $d_{n}=-S_{0}^{-1} S_{n}$, we obtain

$$
h=c+d_{1} h^{2}+d_{2} h^{3}+\ldots
$$

From this formula, we can compute recursively $h_{0}, h_{1}, \ldots$

$$
h_{0}=c, \quad h_{1}=d_{1} c c, \quad h_{2}=d_{1} c d_{1} c c+d_{1} d_{1} c c c+d_{2} c c c, \quad \ldots
$$

Each $d_{i}$ can be interpreted as the symbol of an $(i+1)$-ary operation in Polish notation, as follows

$$
h_{0}=c, \quad h_{1}=d_{1}(c, c), \quad h_{2}=d_{1}\left(c, d_{1}(c, c)\right)+\left(d_{1}\left(d_{1}(c, c), c\right)+d_{2}(c, c, c), \quad \ldots\right.
$$

so that $h$ corresponds to the following sum of ordered trees.

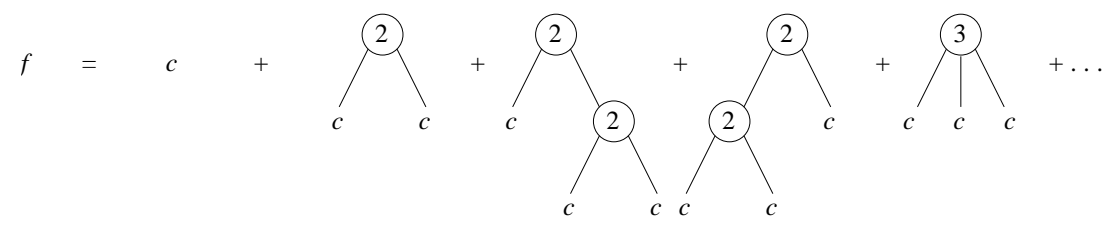

FiguRE 1 . The terms $h_{0}, h_{1}, h_{2}$ expressed as a sum of ordered trees.

Under this interpretation, $h_{n}$ is the sum of all Polish codes of ordered trees with no vertex of arity 1 on $n+1$ leaves. Given such a tree $T$, define its skeleton as the tree obtained by removing the leaves and labeling the internal vertices with their arity. 


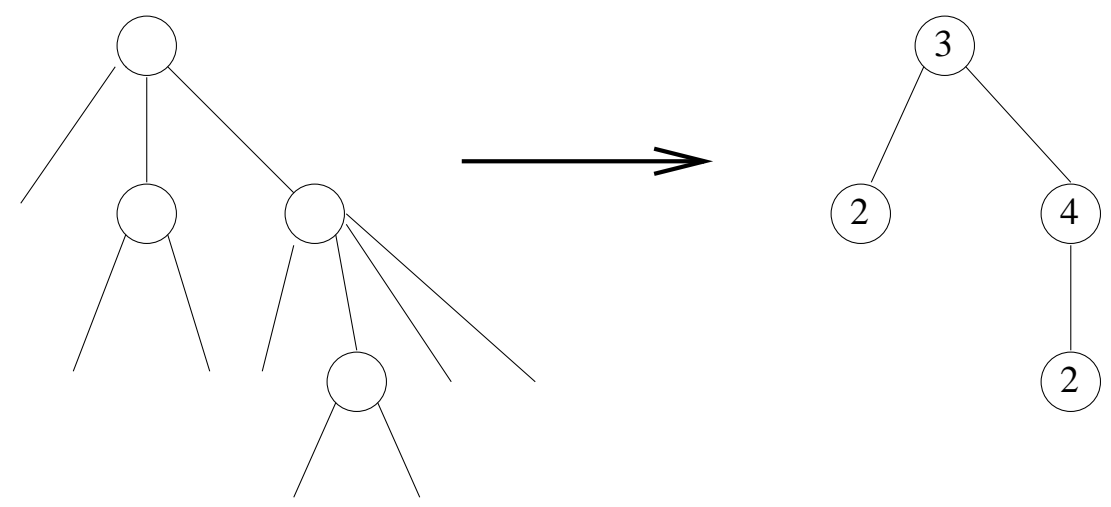

Figure 2. A tree and its skeleton.

Given a skeleton $S$, define its 1 -composition $I_{1}(S)$ as the sequence $\left(j_{1}-1, j_{2}-1, \ldots\right)$, where the $j_{k}$ are the labels of the vertices, given in prefix order.

Novelli and Thibon show that the number of trees with skeleton $S$ is

$$
\prod_{k=1}^{p}\left(\begin{array}{c}
i_{k}+1 \\
a_{k}
\end{array}\right)
$$

where $\left(i_{1}, \ldots, i_{p}\right)=I_{1}(S)$ and $a_{k}$ is the arity of the $k$-th vertex of $S$ in prefix order. Let $I=\left(i_{1}, \ldots, i_{p}\right)$ be a composition of $n$. The coefficient $\lambda_{I}$ of $S^{I}$ in $h_{n}$ is equal to the number of ordered trees on $n+1$ leaves whose sequence of non-zero arities minus one in the prefix reading is $I$. From that, Novelli and Thibon deduce the formula (12), where the set of $\left(a_{1}, \ldots, a_{p-1}\right)$ corresponds to the set of the skeletons of these trees.

\section{The antipode of the one parameter deformation $\mathcal{H}_{\gamma}$ of $\mathcal{H}$}

We shall now give an analogue of formula (12) in $\mathcal{H}_{\gamma}$. For $I \vDash n$, let $\alpha_{I}$ be the coefficient defined by

$$
X_{n}=\sum_{I \models n} \alpha_{I} S^{I}
$$

Our main result is the following theorem:

Theorem 4.1. For any composition I of any integer, the coefficient $\alpha_{I}$ is given by the formula

$$
\alpha_{I}=(-1)^{l(I)} \sum_{\rho \in \mathcal{A}_{l(I)}} \prod_{k=1}^{l(I)}\left(\begin{array}{c}
i_{k} \gamma+1 \\
\rho_{k}
\end{array}\right)
$$

Note that for all $\rho \in \mathcal{A}_{l(I)}$, one has $\rho_{l(I)}=0$, so that the last factor in each term of this formula is 1 , and $\alpha_{I}$ does not depend on the last element of $I$. The rest of this section is devoted to the proof of this formula. 
4.1. A recurrence formula for $X_{n}$. A calculation similar to the one leading to formula (16) yields

$$
1=\sum_{k \geq 0} S_{k}\left(1+X_{1}+X_{2}+\ldots\right)^{k \gamma+1}
$$

Let us set $Y=X-1$. Since one has

$$
\left.X^{k \gamma+1}=(Y+1)^{k \gamma+1}=1+(k \gamma+1) Y+\left(\begin{array}{c}
k \gamma+1 \\
2
\end{array}\right) Y^{2}+\cdots\right),
$$

one can deduce

$$
\begin{aligned}
1 & =\sum_{k \geq 0} S_{k}\left(1+(k \gamma+1) X_{1}+\left(k_{\gamma}+1\right) X_{2}+\ldots\right. \\
& +\left(\begin{array}{c}
k \gamma+1 \\
2
\end{array}\right) X_{1} X_{1}+\left(\begin{array}{c}
k \gamma+1 \\
2
\end{array}\right) X_{1} X_{2}+\left(\begin{array}{c}
k \gamma+1 \\
2
\end{array}\right) X_{2} X_{1}+\ldots \\
& +\ldots
\end{aligned}
$$

This equality can be rewritten

$$
1=S_{0}\left(1+X_{1}+X_{2}+\ldots\right)+\sum_{k \geq 1}\left(S_{k} \sum_{I}\left(\begin{array}{c}
k \gamma+1 \\
l(I)
\end{array}\right) X^{I}\right)
$$

(where the second sum is taken over all the compositions of all the positive integers).

Now, let us extract the homogeneous component of degree $n$. We obtain

$$
0=X_{n}+\sum_{k=1}^{n} S_{k}\left(\sum_{I \vDash n-k}\left(\begin{array}{c}
k \gamma+1 \\
l(I)
\end{array}\right) X^{I}\right)
$$

that is,

$$
X_{n}=-\sum_{k=1}^{n} \sum_{I \models n-k}\left(\begin{array}{c}
k \gamma+1 \\
l(I)
\end{array}\right) S_{k} X^{I}
$$

4.2. Preliminary lemmas. We shall need the following lemmas.

Lemma 4.2. Let I be any composition, and let $\alpha_{I}$ be the coefficient defined in (22). Then,

$$
\alpha_{I}=-\sum_{K \models l(I)-1}\left(\begin{array}{c}
i_{1} \gamma+1 \\
l(K)
\end{array}\right) \alpha_{\left(i_{2}, \ldots, i_{1+k_{1}}\right)} \alpha_{\left(i_{2+k_{1}}, \ldots, i_{1+k_{1}+k_{2}}\right)} \ldots
$$

Proof. One has

$$
\alpha_{I} S^{I}=X_{n \mid I}=-\left(\sum_{k=1}^{n} \sum_{J \models n-k}\left(\begin{array}{c}
k \gamma+1 \\
l(J)
\end{array}\right) S_{k} X^{J}\right)_{\mid I}
$$


Note that a term of the first sum can give a nonzero contribution only if $k=i_{1}$, so that we have

$$
\alpha_{I} S^{I}=-\left(\sum_{J \models n-i_{1}}\left(\begin{array}{c}
i_{1} \gamma+1 \\
l(J)
\end{array}\right) S_{i_{1}} X^{J}\right)_{\mid I}
$$

Set $\tilde{I}=I \backslash\left(i_{1}\right)=\left(i_{2}, i_{3}, \ldots\right)$. This formula becomes

$$
\alpha_{I} S^{\tilde{I}}=-\left(\sum_{|J|=|\tilde{I}|}\left(\begin{array}{c}
i_{1} \gamma+1 \\
l(J)
\end{array}\right) X^{J}\right)_{\mid \tilde{I}}
$$

The term of $\tilde{I}$ in a $X^{J}$ is nonzero only if $J \in \mathfrak{C}(\tilde{I})$, hence, only if there exists $K \vDash l(I)-1$ such that $J=C_{K}(\tilde{I})$.

$$
\alpha_{I} S^{\tilde{I}}=-\left(\sum_{K \models l(I)-1}\left(\begin{array}{c}
i_{1} \gamma+1 \\
l(K)
\end{array}\right) X^{C_{K}(\tilde{I})}\right)_{\mid \tilde{I}}
$$

On another hand,

$$
X^{C_{K}(\tilde{I})}=X_{i_{2}+\ldots+i_{1+k_{1}}} X_{i_{2+k_{1}}+\ldots+i_{1+k_{1}+k_{2}}} \ldots
$$

so that,

$$
\left(X^{C_{K}(\tilde{I})}\right)_{\mid \tilde{I}}=\alpha_{i_{2}, \ldots, i_{1+k_{1}}} \alpha_{i_{2+k_{1}}, \ldots, i_{1+k_{1}+k_{2}}} \ldots S^{\tilde{I}}
$$

From (34) and (36), one deduces the result of the lemma.

Before introducing a second lemma, we shall need the following definition.

Definition 4.3. Set $\mathcal{B}_{1}=\{0\}$. Define by induction $\mathcal{B}_{n}$ as the set of sequences of $n$ integers $\left(\rho_{1}, \ldots, \rho_{n}\right)$ such that there exists a composition $I \vDash n-1$ of length $\rho_{1}$ satisfying

$$
\left\{\begin{aligned}
\left(\rho_{2}, \ldots, \rho_{i_{1}+1}\right) & \in \mathcal{B}_{i_{1}} \\
\left(\rho_{i_{1}+2}, \ldots, \rho_{1+i_{1}+i_{2}}\right) & \in \mathcal{B}_{i_{2}} \\
& \vdots
\end{aligned}\right.
$$

Now, let us give a first explicit formula for $\alpha_{I}$.

Lemma 4.4. Let I be a composition of any integer. Then,

$$
\alpha_{I}=(-1)^{l(I)} \sum_{\rho \in \mathcal{B}_{l(I)}} \prod_{k=1}^{l(I)}\left(\begin{array}{c}
i_{k} \gamma+1 \\
\rho_{k}
\end{array}\right)
$$

Proof. From (30) we deduce by induction on $l(I)$

$$
\alpha_{I}=(-1)^{l(I)} \sum\left(\begin{array}{c}
i_{1} \gamma+1 \\
l(K)
\end{array}\right)\left(\begin{array}{c}
i_{2} \gamma+1 \\
\rho^{(1)}
\end{array}\right)\left(\begin{array}{c}
i_{3} \gamma+1 \\
\rho^{(2)}
\end{array}\right) \ldots\left(\begin{array}{c}
i_{2+k_{1}} \gamma+1 \\
\rho^{(1)}
\end{array}\right)\left(\begin{array}{c}
i_{3+k_{1}} \gamma+1 \\
\rho^{(2)}
\end{array}\right) \ldots
$$


where the sum is taken over the set of sequences $\left(K, \rho^{(1)}, \rho^{(2)}, \ldots\right)$ such that $K \vDash$ $l(I)-1$ and $\rho^{(s)} \in \mathcal{B}_{k_{s}}$ for all $s$.

From this equality, we deduce our result.

To finish the proof of the theorem, we only have to show that for all $n, \mathcal{A}_{n}=\mathcal{B}_{n}$. Indeed, replacing $\mathcal{B}_{l(I)}$ by $\mathcal{A}_{l(I)}$ in (37), we obtain precisely the statement of Theorem 4.1. Since one has $\mathcal{A}_{1}=\mathcal{B}_{1}$, let us fix $n$ and suppose that $\mathcal{A}_{k}=\mathcal{B}_{k}$ for all $k<n$, in order to derive this result by induction.

4.3. Proof that $\mathcal{B}_{n} \subseteq \mathcal{A}_{n}$. Let $\rho \in \mathcal{B}_{n}$. Then, there exists a composition $I \vDash n-1$ of length $\rho_{1}$ such that $\rho$ and $I$ verify the relations given in definition 4.3.

Let us rewrite $\rho$ in the form $\left(\rho_{1}, \rho^{(1)}, \rho^{(2)}, \ldots\right)$, where for all $s, \rho^{(s)} \in \mathcal{B}_{i_{s}}$. By assumption, one has $\mathcal{B}_{i_{s}}=\mathcal{A}_{i_{s}}$. Hence,

$$
\rho_{1}+\ldots+\rho_{n}=l(I)+\left(i_{1}-1\right)+\left(i_{2}-1\right)+\ldots=n-1
$$

Let $s$ be such that $2 \leq s \leq n$. Hence, $\rho_{s}$ will be the $j$ th element of some $\rho^{(k)} \in \mathcal{B}_{i_{k}}$, and one will have

$$
s=n+j-i_{k+1}-i_{k+2}-\ldots
$$

Since $\rho^{(k)} \in \mathcal{B}_{i_{k}}=\mathcal{A}_{i_{k}}$, one will have also

$$
\rho_{s}+\ldots+\rho_{1+i_{1}+i_{2}+\ldots+i_{k}} \leq i_{k}-j
$$

Moreover,

$$
\rho^{(k+1)} \in \mathcal{B}_{i_{k+1}}=\mathcal{A}_{i_{k+1}}, \quad \rho^{(k+2)} \in \mathcal{B}_{i_{k+2}}=\mathcal{A}_{i_{k+2}}, \quad \ldots,
$$

so that

$$
\begin{aligned}
\rho_{s}+\ldots+\rho_{n} & \leq i_{k}-j+\left(i_{k+1}-1\right)+\left(i_{k+1}-2\right)+\ldots \\
& \leq i_{k}-j+i_{k+1}+i_{k+2}+\ldots \\
& \leq n-s
\end{aligned}
$$

Hence, one has $\rho \in \mathcal{A}_{n}$, so that

$$
\mathcal{B}_{n} \subseteq \mathcal{A}_{n}
$$

Example 4.5. Suppose that $\mathcal{B}_{2}=\mathcal{A}_{2}$ and let us consider the sequence $\rho=(2,1,0,1,0)$. It belongs to $\mathcal{B}_{5}$, because $(1,0) \in \mathcal{A}_{2}=\mathcal{B}_{2}$.

$$
\begin{array}{|c|c|c|c|c|}
\multicolumn{1}{c}{\leq 1 \leq 0} & \leq 1 \leq 0 \\
\hline 2 & 1 & 0
\end{array} \quad \begin{array}{|c|c|}
\hline 1 & 0 \\
\hline
\end{array}
$$

On the top of each box, we have written a number greater than the sum of its value and the values of all the cells on the right in its block. In this way, we can also write

$$
\begin{array}{|l|c|c|c|c|}
4 & \leq 2 \leq 1 \leq 1 \leq 0 \\
\hline 2 & 1 & 0 & 1 & 0 \\
\hline
\end{array}
$$

and

$\begin{aligned} & 4 \\
& 4\end{aligned} \quad 3 \leq 2 \leq 1 \leq 0$
\begin{tabular}{|c|c|c|c|c}
\hline 2 & 1 & 0 & 1 & 0 \\
\hline
\end{tabular}


so that we have $\rho \in \mathcal{A}_{5}$.

4.4. Proof that $\mathcal{A}_{n} \subseteq \mathcal{B}_{n}$. Let $\rho \in \mathcal{A}_{n}$, and suppose that

$$
\rho=\left(\rho_{1}, \rho^{(1)}, \rho^{(2)}, \ldots\right)
$$

where all the $\rho^{(s)}$ except the last one belong to a $\mathcal{B}_{i_{s}}=\mathcal{A}_{i_{s}}$. Let this last one be $\lambda$. All the inequalities necessary to have $\lambda \in \mathcal{A}_{l(\lambda)}$ are satisfied, since $\rho \in \mathcal{A}_{n}$. The only problem we can encounter is to have $|\lambda| \neq l(\lambda)-1$. On another hand, one has

$$
|\lambda| \leq l(\lambda)-1
$$

since $\rho \in \mathcal{A}_{n}$. In the case where $|\lambda|<l(\lambda)-1$, suppose that for all $j$, one could not build an element of $\mathcal{A}_{j}$ with the $j$ first terms of $\lambda$, so that we would have

$$
\begin{array}{ll} 
& \left(\lambda_{1} \neq 0\right) \\
\text { and } & \left(\lambda_{1}+\lambda_{2} \neq 1 \text { or } \lambda_{2}>0\right) \\
\text { and } & \left(\lambda_{1}+\lambda_{2}+\lambda_{3} \neq 2 \text { or } \lambda_{2}+\lambda_{3}>1 \text { or } \lambda_{3}>0\right) \\
\text { and } & \ldots
\end{array}
$$

Now, let us derive the following lemma.

Lemma 4.6. Conditions (50) imply for all $j$ the inequality

$$
\lambda_{1}+\lambda_{2}+\ldots+\lambda_{j}>j-1
$$

Proof. Under conditions (50), one has $\lambda_{1} \neq 0$, so that $\lambda_{1}>0$. In order to derive our result by induction on $j$, let us fix $j$ and suppose that it is true when one replaces $j$ by any $k<j$. Looking at (50), one can see that there are two distinct cases.

1st case :

$$
\lambda_{1}+\ldots+\lambda_{j} \neq j-1
$$

In this case, since one has by assumption

$$
\lambda_{1}+\ldots+\lambda_{j-1}>j-2,
$$

a first possibility is $\lambda_{1}+\ldots+\lambda_{j-1}=j-1$. In this case, when $\lambda_{j}=0$, one has $\lambda_{1}+\ldots+\lambda_{j}=j-1$. Since this statement is in contradiction with our hypothesis, we must have $\lambda_{j}>0$, and inequality (51) is true.

The only other possibility is that $\lambda_{1}+\ldots+\lambda_{j-1}>j-1$, in which case inequality (51) is also true.

2nd case : there exists an integer $k$ such that $2 \leq k \leq j$ and

$$
\lambda_{k}+\ldots+\lambda_{j}>j-k
$$

One has by assumption

$$
\lambda_{1}+\ldots+\lambda_{k-1}>k-2,
$$

Since the $\lambda_{i}$ are integers, one can rewrite (54) and (55) as follows.

$$
\lambda_{k}+\ldots+\lambda_{j} \geq j-k+1
$$


and

$$
\lambda_{1}+\ldots+\lambda_{k-1} \geq k-1
$$

so that,

$$
\lambda_{1}+\ldots+\lambda_{j} \geq j
$$

and inequality (51) is again true, so that it is true in all cases.

By setting $j=l(\lambda)$ in (51), we obtain

$$
|\lambda|>l(\lambda)-1
$$

This is in contradiction with (49), so that we have shown that one can build from the sequence $\left(\rho_{1}, \rho^{(1)}, \ldots, \rho^{(k)}\right)$ a $\rho^{(k+1)}$ such that a $i_{k+1}$ satifying

$$
\rho^{(k+1)} \in \mathcal{B}_{i_{k+1}}
$$

does exist. By repeating of this process, one can build a sequence

$$
\left(\rho_{1}, \rho_{1}^{(1)}, \rho_{2}^{(1)}, \ldots, \rho_{1}^{(2)}, \rho_{2}^{(2)}, \ldots\right)=\rho
$$

such that for all $s$, there exists a $i_{s}$ verifying $\rho^{(s)} \in \mathcal{B}_{i_{s}}$. Note that we do have $I \vDash l(\rho)-1$, and that (39) implies $\rho_{1}=l(I)$. Hence, $\rho \in \mathcal{B}_{n}, \mathcal{A}_{n} \subseteq \mathcal{B}_{n}$, so that we have derived the property

$$
\mathcal{A}_{n}=\mathcal{B}_{n}
$$

Hence, the result of lemma 4.4 is identical with the one of theorem 4.1.

4.5. Remarks and combinatorial interpretation. Subtracting each row to the first one, we can rewrite the system (9) as

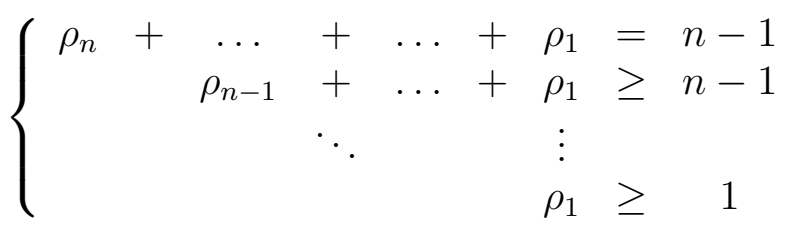

This remark allows us to see that formula (23) is consistent with formula (12), which corresponds to the case $\gamma=1$. Note also that the set $\mathcal{A}_{n}$ is in bijection with the set of nondecreasing parking functions of length $n$. An explicit bijection can be obtained by associating $\left(\rho_{1}, \rho_{2}, \ldots\right) \in \mathcal{A}_{n}$ with the parking function

$$
(\underbrace{1, \ldots, 1}_{\rho_{1} \text { times }}, \underbrace{2, \ldots, 2}_{\rho_{2} \text { times }}, \ldots) \text {. }
$$

Hence, it is clear that $\mathcal{A}_{n}$ is a Catalan set, which is also consistent with results of Novelli and Thibon. For any tree $T$, denote by $a_{k}(T)$ the arity of the $k$ th vertex of the skeleton of $T$, and for any $x$ and $i$, set

$$
x^{i \downarrow}=x(x-1)(x-2) \ldots(x-i+1)
$$


From the combinatorial interpretation of (12), we can rewrite the formula (23) as

$$
\alpha_{I}=(-1)^{l(I)} \sum_{T} \prod_{k=1}^{l(I)} \frac{\left(i_{k} \gamma+1\right)^{a_{k}(T) \downarrow}}{\left(i_{k}+1\right)^{a_{k}(T) \downarrow}}
$$

where the sum is over the set of ordered trees $T$ on $n+1$ leaves whose sequence of non-zero arities minus one in prefix reading is $I$.

\section{Other Closed formulas for some COEFFiCients in EXPANSions of $X_{n}$}

In this section, we will be interested in the coefficients $\beta_{I}$ in

$$
X_{n}=\sum_{I} \beta_{I} R_{I}
$$

where $\left(R_{I}\right)$ denotes the basis of ribbon noncommutative symmetric functions.

We can give a first expression of the $\beta_{I}$ by expanding the $S^{J}$ over the $R_{I}$, that is

$$
\beta_{I}=\sum_{J \leq R} \alpha_{J}
$$

where we use the notation $J \leq_{R} I$ to see that $J$ is a refinement of $I$, that is, for some $K \vDash l(J)$,

$$
\left\{\begin{array}{l}
j_{1}+j_{2}+\ldots+j_{k_{1}}=i_{1} \\
j_{k_{1}+1}+\ldots+j_{k_{1}+k_{2}}=i_{2} \\
\vdots
\end{array}\right.
$$

5.1. A closed formula for $\beta_{1^{n}}$. Denote by $\delta_{n}$ the coefficient $\beta_{1^{n}}$ for any $n$. From (68), we deduce that this coefficient is also equal to $\alpha_{1^{n}}$, so that from (30) we have

$$
\delta_{n}=-\sum_{I \vDash n-1}\left(\begin{array}{c}
\gamma+1 \\
l(I)
\end{array}\right) \prod_{i \in I} \delta_{I}
$$

From that, the series $L(t)=\sum_{n \geq 0} \delta_{n} t^{n}$ can be rewritten

$$
L(t)=1-t \sum_{n \geq 1} \sum_{I \models n-1}\left(\begin{array}{c}
\gamma+1 \\
l(I)
\end{array}\right) \prod_{i \in I}\left(\delta_{i} t^{i}\right)
$$

so that

$$
L(t)=1-t \sum_{I}\left(\begin{array}{c}
\gamma+1 \\
l(I)
\end{array}\right) \prod_{i \in I} \delta_{i} t^{i}
$$

where the sum is over all the compositions $I$. By setting $\tilde{L}(t)=L(t)-1$, we then obtain

$$
L(t)=1-t\left(\left(\begin{array}{c}
\gamma+1 \\
0
\end{array}\right)+\left(\begin{array}{c}
\gamma+1 \\
1
\end{array}\right) \tilde{L}(t)+\left(\begin{array}{c}
\gamma+1 \\
2
\end{array}\right) \tilde{L}(t)^{2}+\ldots\right)
$$

so that

$$
L(t)=1-t L(t)^{\gamma+1}
$$


Then, we have $1-L(t)=t L(t)^{\gamma+1}$, so that the series $y(t)=1-L(t)$ is a solution of the equation

$$
t=\frac{y(t)}{(1-y(t))^{\gamma+1}}=y \sigma_{y}(\gamma+1)
$$

(Recall that the complete homogeneous symmetric functions of a scalar $\alpha$ are defined by $\left.\sigma_{t}(\alpha)=\sum_{n \geq 0} h_{n}(\alpha) t^{n}=\frac{1}{(1-t)^{\alpha}}\right)$. From the classical Lagrange inversion formula (see [9], Ex. 24 p. 35), we can write

$$
y(t)=t \sigma_{t}^{\star}(\gamma+1)
$$

where $F \mapsto F^{\star}$ is here the antipode of the Faá di Bruno algebra, that is, the one of the classical commutative case with $\gamma=1$. From that, we deduce that for $n>0$,

$$
-\lambda_{n}=h_{n-1}^{\star}(\gamma+1) \text {, }
$$

that is,

$$
\begin{aligned}
\lambda_{n} & =-\frac{1}{n} h_{n-1}(-n(\gamma+1)) \\
& =-\frac{1}{n}\left(\begin{array}{c}
-n(\gamma+1)+(n-1)-1 \\
n-1
\end{array}\right) \\
& =-\frac{1}{n}\left(\begin{array}{c}
-n \gamma-2 \\
n-1
\end{array}\right)
\end{aligned}
$$

On another hand, we have

$$
\left(\begin{array}{c}
-n \gamma-2 \\
n-1
\end{array}\right)=\frac{(-n \gamma-2)(-n \gamma-3) \ldots(-n \gamma-n)}{(n-1) !}=(-1)^{n-1}\left(\begin{array}{c}
n(\gamma+1) \\
n-1
\end{array}\right)
$$

Summarizing, we have shown the following proposition.

Proposition 5.1. The coefficient $\beta_{1^{n}}$ is given by

$$
\beta_{1^{n}}=(-1)^{n} \frac{1}{n}\left(\begin{array}{c}
n(\gamma+1) \\
n-1
\end{array}\right)
$$

5.2. A closed formula for $\beta_{n}$. We have from (68)

$$
\beta_{n}=\sum_{I \models n} \alpha_{I}
$$

From (30), this equality can be rewritten as

$$
\beta_{n}=-\sum_{I \models n} \sum_{K \models l(I)-1}\left(\begin{array}{c}
i_{1} \gamma+1 \\
l(K)
\end{array}\right) \alpha_{\left(i_{2}, \ldots, i_{1+k_{1}}\right)} \alpha_{\left(i_{2+k_{1}}, \ldots, i_{1+k_{1}+k_{2}}\right)} \ldots
$$

By setting

$$
J=\left(i_{1}, i_{2}+\ldots+i_{1+k_{1}}, i_{2+k_{1}}+\ldots+i_{1+k_{1}+k_{2}}, \ldots\right),
$$

we can deduce

$$
\beta_{n}=-\sum_{J \models n}\left(\begin{array}{c}
j_{1} \gamma+1 \\
l(J)-1
\end{array}\right) \sum_{J^{(2)} \models j_{2}, J^{(3)} \models j_{3}, \ldots} \alpha_{J^{(2)}} \alpha_{J^{(3)}} \ldots
$$


From (68) and this equation we have

$$
\beta_{n}=-\sum_{J \models n}\left(\begin{array}{c}
j_{1} \gamma+1 \\
l(J)-1
\end{array}\right) \beta_{j_{2}} \beta_{j_{3}} \ldots
$$

Now, let us set

$$
\beta(t)=\sum_{n \geq 1} \beta_{n} t^{n}
$$

We then obtain

$$
\begin{aligned}
\beta(t) & =-\sum_{r \geq 1} \sum_{l(I)=r}\left(\begin{array}{c}
i_{1} \gamma+1 \\
r-1
\end{array}\right) t^{i_{1}} \beta_{i_{2}} t^{i_{2}} \beta_{i_{3}} t^{i_{3}} \ldots \beta_{i_{r}} t^{i_{r}} \\
& =-\sum_{i_{1} \geq 1, r \geq 1}\left(\begin{array}{c}
i_{1} \gamma+1 \\
r-1
\end{array}\right) t^{i_{1}} \beta(t)^{r-1} \\
& =-\sum_{k \geq 1, s \geq 0} t^{k}\left(\begin{array}{c}
k \gamma+1 \\
s
\end{array}\right) \beta(t)^{s} \\
& =-\sum_{k \geq 1} t^{k}\left(\sum_{s \geq 0}\left(\begin{array}{c}
k \gamma+1 \\
s
\end{array}\right) \beta(t)^{s}\right) \\
& =-\sum_{k \geq 1} t^{k}(1+\beta(t))^{k \gamma+1} \\
& =-(1+\beta(t)) \sum_{k \geq 1}\left(t(1+\beta(t))^{\gamma}\right)^{k},
\end{aligned}
$$

so that,

$$
\beta(t)=-\frac{(1+\beta(t)) t(1+\beta(t))^{\gamma}}{1-t(1+\beta(t))^{\gamma}}
$$

This equality can be rewritten as

$$
\beta(t)-t \beta(t)(1+\beta(t))^{\gamma}=-t(1+\beta(t))^{\gamma}-t \beta(t)(1+\beta(t))^{\gamma}
$$

We then have $\beta(t)=-t(1+\beta(t))^{\gamma}$, so that the series

$$
B(t)=\sum_{n \geq 0} \beta_{n} t^{n}=1+\beta(t)
$$

is a solution of

$$
B(t)=1-t B(t)^{\gamma}
$$

This equation is exactly the one of $L(t)$, where $\gamma+1$ is replaced by $\gamma$. From that, we can give the following proposition.

Proposition 5.2. The coefficient $\beta_{n}$ is given by

$$
\beta_{n}=(-1)^{n} \frac{1}{n}\left(\begin{array}{c}
n \gamma \\
n-1
\end{array}\right)
$$




\section{A ReCURRENCE Formula for the $\beta_{I}$}

The coefficients $\beta_{I}$ satisfy some relations. For example, let us give the following proposition.

Proposition 6.1. Let $J$ be a composition of length $s$ whose last part $j_{s}$ satisfies $j_{s}>1$. Then,

$$
\beta_{J}=\beta_{\left(j_{1}, \ldots, j_{s-1}, j_{s}-1,1\right)}+\beta_{\left(j_{1}, \ldots, j_{s-1}, j_{s}-1\right)}
$$

Proof. Denote by $\tilde{J}$ the composition obtained by subtracting 1 to the last part of $J$, and by $\hat{J}$ the one obtained by adding a part 1 at the end of $\tilde{J}$. Then, formula (68) allows us to write

$$
\beta_{J}=\sum_{K \leq_{R} \hat{J}} \alpha_{K}+\sum_{K \leq_{R} \tilde{J}} \alpha_{K^{\prime}}
$$

(where $K^{\prime}$ is defined as the composition obtained by adding 1 to the last part of $K$ ). Indeed, the refinements of $J$ whose last part is 1 are exactly the refinements of $\tilde{J}$ with a part 1 more added at the end, that is, the refinements of $\hat{J}$. On another hand, the refinements of $J$ whose last part is not 1 are exactly the refinements of $\tilde{J}$ with last part incremented of 1 . Since the $\alpha_{I}$ do not depend on the last part of $I$, the equation (101) can be rewritten as

$$
\beta_{J}=\sum_{K \leq_{R} \hat{J}} \alpha_{K}+\sum_{K \leq_{R} \tilde{J}} \alpha_{K}
$$

And from (68) we deduce

$$
\beta_{J}=\beta_{\hat{J}}+\beta_{\tilde{J}}
$$

We also give in this section a recurrence formula for the coefficients $\beta_{I}$. Namely, we express $\beta_{I}$ in terms of the $\beta_{J}$ with $|J|<|I|$. In order to do that, let us introduce some preliminary definitions.

\subsection{Cuts of a composition.}

Definition 6.2. Let $n>0$ be an integer, and $\phi$ a function from the set of the $n$ first nonzero integers to itself. We will say that $\phi$ is a cut function of size $n$ if

$$
\phi(1)=1
$$

and

$$
0 \leq \phi(k+1)-\phi(k) \leq 1
$$

for any $k$ such that this equality makes sense. 
Definition 6.3. For all compositions $J$ and for all cut functions $\phi$ of $l(J)$, let us define the cut of $J$ corresponding to $\phi$ as the following sequence of compositions :

$$
D_{J, \phi}=\left(J^{(1)}, J^{(2)}, \ldots\right) \text {, }
$$

where for all $n, J^{(n)}$ is defined by

$$
J^{(n)}=\left(j_{k}, j_{k+1}, j_{k+2}, \ldots, j_{s}\right)
$$

where $k$ and $s$ are such that $\phi(x)=n$ for all integers $x \in[k, s]$, and $\phi(x) \neq n$ for all other values of $x$, so that

$$
\phi(k)=\phi(k+1)=\ldots=\phi(s)=n
$$

We also define the length of a cut as the number of compositions of which the cut is composed. Finally, we will say that a cut $D_{J, \phi}$ is a refinement of a cut $D_{\tilde{J}, \tilde{\phi}}$ when each $J^{(k)}$ from the first cut is a refinement of the $\tilde{J}^{(k)}$ corresponding in the second one, that is, we will write

$$
\left(J^{(1)}, J^{(2)}, \ldots\right) \leq_{R}\left(\tilde{J}^{(1)}, \tilde{J}^{(2)}, \ldots\right)
$$

when

$$
J^{(1)} \leq_{R} \tilde{J}^{(1)}, J^{(2)} \leq_{R} \tilde{J}^{(2)}, \cdots
$$

We shall also need the following definitions.

Definition 6.4. Let $I$ be a composition and $J$ a refinement of $I$. We then define the I-cut of $J$ as the cut

$$
\left(J^{(1)}, J^{(2)}, \ldots\right)
$$

such that for all $k, J^{(k)} \vDash i_{k}$. We also define $\phi_{I, J}$ as the corresponding cut function. Note that it is such that for all $s$,

$$
\sum_{\phi_{I, J}(k)=s} j_{k}=i_{s}
$$

Definition 6.5. Let $I$ and $J$ be two compositions of same weight $n$ such that

$$
J \leq_{R} I
$$

We will say that a cut $D_{\psi, J}$ is I-admissible and that the corresponding cut function $\psi$ is $(I, J)$-admissible if

$$
\psi(2)=2
$$

and if for all $k$ and $s$ such that $\phi_{I, J}(k)=\phi_{I, J}(s)$,

$$
\psi(k) \neq \psi(s)
$$

For example, if $J \neq I$, the $I$-cut of $J$ is not I-admissible

Now, let $J$ and $I$ be two compositions such that

$$
J \leq_{R} I
$$

and let

$$
D_{J, \phi}=\left(J_{\phi}^{(1)}, J_{\phi}^{(2)}, \ldots\right)
$$


be any cut of $J$ such that

$$
\phi(2)=2
$$

(or, equivalently, $J_{\phi}^{(1)}=\left(j_{1}\right)$ ). Then, we can obtain from $D_{J, \phi}$ a $I$-admissible cut

$$
D_{\tilde{J}, \tilde{\phi}}=\mathcal{N}\left(D_{J, \phi}\right)
$$

by replacing each $J_{\phi}^{(k)}=\left(j_{x}, j_{x+1}, \ldots, j_{y}\right)$ by the composition in which each part is the sum of all the $j_{s}$ corresponding to values of $s$ such that $x \leq s \leq y$ and such that all the $\phi_{I, J}(s)$ are equal to the same $n$. The parts of this $\tilde{J}_{\tilde{\phi}}^{(k)}$ must be arranged by increasing order of the corresponding $n$ for all possible $\mathrm{n}$. This new cut is $I$-admissible, and for all $k$, one has

$$
J_{\phi}^{(k)} \leq_{R} \tilde{J}_{\tilde{\phi}}^{(k)}
$$

so that $D_{J, \phi}$ is a refinement of $D_{\tilde{J}, \tilde{\phi}}$. Moreover, the set

$$
\mathcal{D}_{\tilde{J}, \tilde{\phi}}=\left\{D_{J, \phi} / \phi(2)=2 \text { and } D_{\tilde{J}, \tilde{\phi}}=\mathcal{N}\left(D_{J, \phi}\right)\right\}
$$

coincides with the set of the refinements of $D_{\tilde{J}, \tilde{\phi}}$ such that

$$
J^{(1)}=\left(j_{1}\right)
$$

6.2. Expression of $\beta_{I}$ in function of the $\beta_{J}$ with $|J|<|I|$. Formula (30) can be rewritten as

$$
\alpha_{J}=-\sum_{D_{J, \phi}}\left(\begin{array}{c}
j_{1} \gamma+1 \\
l\left(D_{J, \phi}\right)-1
\end{array}\right) \alpha_{J_{\phi}^{(2)}} \alpha_{J_{\phi}^{(3)}} \ldots \alpha_{J_{\phi}^{\left(l\left(D_{J, \phi}\right)\right)}}
$$

where the sum is over all the cuts $D_{J, \phi}$ of $J$ verifying

$$
\phi(2)=2
$$

We then deduce from (68) the following formula:

$$
\beta_{I}=-\sum_{D_{J, \phi}, J \leq_{R} I}\left(\begin{array}{c}
j_{1} \gamma+1 \\
l\left(D_{J, \phi}\right)-1
\end{array}\right) \alpha_{J_{\phi}^{(2)}} \alpha_{J_{\phi}^{(3)}} \ldots \alpha_{J_{\phi}^{\left(l\left(D_{J, \phi}\right)\right)}}
$$

where this time, the sum is over all the cuts $D_{J, \phi}$ of all the refinements $J$ of $I$ that satisfy (124). By setting

$$
D_{\tilde{J}, \tilde{\phi}}=\mathcal{N}\left(D_{J, \phi}\right)
$$

we then obtain

$$
\beta_{I}=-\sum_{J \leq R}\left(\begin{array}{c}
j_{1} \gamma+1 \\
l\left(D_{J, \phi}\right)-1
\end{array}\right) \alpha_{J_{\phi}^{(2)}, \tilde{\phi}, D_{J, \phi} \in \mathcal{D}_{\tilde{J}, \tilde{\phi}}} \alpha_{J_{\phi}^{(3)}} \ldots \alpha_{J_{\phi}^{\left(l\left(D_{J, \phi}\right)\right)}}
$$

where the sum is over all the cuts of all the refinements of $I$ that belong to a $\mathcal{D}_{\tilde{J}, \tilde{\phi}}$ for certain $I$-admissible cut $D_{\tilde{I}, \tilde{\phi}}$. In other words, it is over all the refinements of all the $I$-admissible cuts whose first element has only one part. By setting $K=\tilde{J}$, we then deduce from (68) the following proposition. 
Proposition 6.6. The coefficients $\beta_{I}$ satisfy the following recurrence formula:

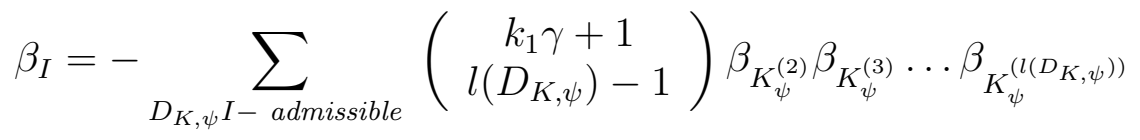

6.3. Expression of $\beta_{I}$ when $I$ has two parts. In this section, we suppose that

$$
I=\left(i_{1}, i_{2}\right)
$$

In this case, for any $J \leq_{R} I$, the $I$-cut of $J$ is $\left(J^{(1)}, J^{(2)}\right)$ with

$$
J^{(1)} \vDash i_{1} \text { et } J^{(2)} \vDash i_{2}
$$

Then, the $I$-admissible cuts of $J$ are the ones with one of these two types :

$$
\left(\left(j_{1}\right),\left(j_{2}\right), \ldots,\left(j_{l(J)}\right)\right)
$$

or

$$
\left(\left(j_{1}^{(1)}\right),\left(j_{2}^{(1)}\right), \ldots,\left(j_{l\left(J^{(1)}\right)}^{(1)}, j_{1}^{(2)}\right),\left(j_{2}^{(2)}\right), \ldots\right)
$$

The formula (128) can then be rewritten in this case as

$$
\begin{aligned}
\beta_{I}= & -\sum_{J \models i_{1}, K \models i_{2}}\left(\begin{array}{c}
j_{1} \gamma+1 \\
l(J)+l(K)-1
\end{array}\right) \beta_{j_{2}} \beta_{j_{3}} \ldots \beta_{j_{l(J)}} \beta_{k_{1}} \beta_{k_{2}} \ldots \beta_{k_{l(K)}} \\
& -\sum_{J \models i_{1}, K \models i_{2}}\left(\begin{array}{c}
j_{1} \gamma+1 \\
l(J)+l(K)-2
\end{array}\right) \beta_{j_{2}} \beta_{j_{3}} \ldots \beta_{\left(j_{l(J)}, k_{1}\right)} \beta_{k_{2}} \ldots \beta_{k_{l(K)}}
\end{aligned}
$$

From that, we deduce the result of the following proposition.

Proposition 6.7. The coefficient $\beta_{I}$ corresponding to a composition $I=\left(i_{1}, i_{2}\right)$ with two parts satisfies the recurrence formula

$$
\begin{aligned}
\beta_{I}= & -\sum_{J \models i_{1}, K \models i_{2}}\left(\begin{array}{c}
j_{1} \gamma+1 \\
l(J)+l(K)-1
\end{array}\right)\left(\prod_{j \in \tilde{J}} \beta_{j}\right)\left(\prod_{k \in K} \beta_{k}\right) \\
& -\sum_{0<|J|<i_{1}, 0 \leq|K|<i_{2}}\left(\begin{array}{c}
j_{1} \gamma+1 \\
l(J)+l(K)
\end{array}\right)\left(\prod_{j \in \tilde{J}} \beta_{j}\right)\left(\prod_{k \in K} \beta_{k}\right) \beta_{\left(i_{1}-|J|, i_{2}-|K|\right)}
\end{aligned}
$$

where we have made use of the notation

$$
\tilde{J}=\left(j_{2}, j_{3}, \ldots, j_{l(J)}\right)
$$

\section{REFERENCES}

[1] C. Brouder, A. Frabetti and C. Krattenthaler, Non-commutative Hopf algebra of formal diffeomorphisms, QA/0406117, 2004 - arxiv.org

[2] L. Foissy, Faà di Bruno subalgebras of the Hopf algebra of planar trees from combinatorial Dyson-Schwinger equations, Adv. Math. 218 (2008), no. 1, 136-162.

[3] I.M. Gelfand, D. Krob, A. Lascoux, B. Leclerc, V. S. Retakh, and J.-Y. Thibon, Noncommutative symmetric functions, Adv. in Math. 112 (1995), 218-348.

[4] I. Gessel, Noncommutative Generalization and q-analog of the Lagrange Inversion Formula, Trans. Amer. Math. Soc. 257 (1980), no. 2, 455-482. 
[5] S. A. Joni and G.-C. Rota, Coalgebras and bialgebras in combinatorics, Contemp. Math. 6 (1982), 1-47.

[6] D. Krob, B. Leclerc and J.-Y. Thibon, Noncommutative symmetric functions II: Transformations of alphabets, Intern. J. Alg. Comput. 7 (1997), 181-264.

[7] A. Lascoux, Symmetric functions and combinatorial operators on polynomials, CBMS Regional Conference Series in Mathematics 99, American Math. Soc., Providence, RI, 2003; xii+268 pp.

[8] C. Lenart, Lagrange inversion and Schur functions, J. Algebraic Combin. 11 (2000), 1, 69-78.

[9] I.G. Macdonald, Symmetric functions and Hall polynomials, 2nd ed., Oxford University Press, 1995.

[10] J.-C Novelli and J.-Y. ThiBon, Noncommutative symmetric functions and Lagrange inversion, Adv. in Appl. Math. 40 (2008), 8-35.

[11] I. Pak, A. Postnikov, and V. S. Retakh, Noncommutative Lagrange Theorem and Inversion Polynomials, preprint, 1995, available at http://www-math.mit.edu/ pak/research.html.

[12] G. N. RAneY, Functional composition patterns and power series reversion, Trans. Amer. Math. Soc. 94 (1960), 441-451.

Institut Gaspard Monge, Université Paris-Est Marne-la-Vallée, 5 Boulevard Descartes, Champs-Sur-Marne, 77454 Marne-la-Vallée Cedex 2, France

E-mail address: bultel@univ-mlv.fr 